\title{
Management of genital herpes by genitourinary physicians: does experience or doctor's gender influence clinical management?
}

\author{
J M Russell, M Cracknell, S E Barton, J Catalan
}

\begin{abstract}
Objective-To determine the variation in management of genital herpes by genitourinary physicians, and whether their duration of experience or gender influence their clinical management.

Methods-A postal questionnaire was sent to UK consultant genitourinary physicians with detailed questions about management of primary and recurrent herpes. The gender and duration of genitourinary medicine experience of the physicians were also recorded.

Results-One hundred and eighty two questionnaires were sent, 112 (62\%) returned. Eighty-one (72\%) physicians treat all patients with primary genital herpes, but physicians with more than 20 years experience were significantly $(p<0.05)$ more likely to treat only "severe" primary attacks. Most experienced physicians were also most likely $(p<0.05)$ to prescribe topical acyclovir. Prescription of suppressive acyclovir was also influenced by the experience of the physician, the least experienced physicians being more likely to prescribe to patients who were HIV antibody positive or to those entering new relationships, whereas the more experienced prescribed to those patients who were particularly anxious $(p<0.05$ for each of these). Male physicians were significantly more likely to agree with the proposition that men cope better with genital herpes $(54 \%)$ than female physicians $(24 \%, p<0.01)$.

Conclusion-The response to the questionnaire illustrates that management of genital herpes is influenced by the duration of the physicians clinical experience. Gender of the physician may have an indirect role to play as we have shown that physicians differ in their perception of how the sexes cope with genital herpes.
\end{abstract}

(Genitourin Med 1993;69:115-118)

\section{Introduction}

Primary and recurrent genital herpes simplex virus (HSV) infections account for about $5 \%$ of genitourinary clinic attendances. The condition causes much distress, particularly in those with frequent recurrences. ${ }^{12}$ At present acyclovir is the only effective anti-viral agent licensed in the United Kingdom for use in both primary attacks and prophylaxis of recurrences. Reports from a patient self-help group, The Herpes Association, have suggested that there is a considerable variation in the management of this condition by genitourinary physicians, especially of patients with recurrent episodes. Genital HSV became notifiable on sexually transmitted disease KC60 returns in 1982 and acyclovir was not licensed until just over 10 years ago, so it is possible that physicians may differ in their views about genital $\mathrm{HSV}$ according to their experience, leading to the development of different treatment strategies.

Acyclovir prescribed prophylactically at a dose of $400 \mathrm{mg}$ twice daily costs $£ 105.95$ (US\$150) per month and this may be an inhibiting factor against physicians prescribing $\mathrm{it}^{3}$ particularly where local restraints on departmental pharmacy budgets exist. Apart from cost, several clinical considerations may affect which patients are offered suppressive therapy, although different physicians may attribute different importance to specific factors, such as frequency of attacks, severity of lesions or the associated anxiety. The personal characteristics of both patient and the physician may also have a role to play in dictating the prescribing pattern. This study was designed to discover whether there was a true variation in the management of genital herpes by genitourinary physicians, and whether these differences were related to any specific characteristics of the prescribing physicians.

\section{Methods}

A postal questionnaire was sent to 182 genitourinary physicians in the United Kingdom, followed by one postal reminder. The questionnaire included structured questions about the management of primary and recurrent herpes, local clinic policy and whether any local financial restraints exist. The questionnaires were anonymous, but personal details including age, gender and duration of genitourinary experience were requested. Results were analysed using the Fishers exact and $\chi^{2}$ tests for group comparisons as appropriate.

\section{Results}

One hundred and twelve questionnaires were returned, a $62 \%$ response rate. Seventy six from male consultants (68\%) and $25(22 \%)$ from female consultants. Eleven respondents $(10 \%)$ did not indicate their sex. One hundred and four indicated their duration of clinical experience in genitourinary medicine: 25 \\ Genitourinary \\ Charing Cross \\ 8 December 1992
}


$(22 \%)$ had less than 10 years experience, 56 $(50 \%)$ between 10 and 20 years, and, 23 $(21 \%)$ more than 20 years service.

\section{Management of primary attack}

The majority of physicians, $81(72 \%)$ treat all patients presenting with primary genital herpes, $23(21 \%)$ treat only those with "severe" primary attacks and eight $(7 \%)$ did not respond to this question. A non-significant difference in this preference was seen between male consultants $20 / 76(26 \%)$ treating only severe attacks, compared with $3 / 25$ (12\%) female consultants doing so. A significant difference $(p<0.05)$ was seen when duration of experience was considered: $8 / 23$ $(35 \%)$ of physicians with more than 20 years experience treat only "severe" attacks compared with $8 / 56(14 \%)$ of those with $10-20$ years experience, and $5 / 25(20 \%)$ with less than 10 years experience.

The acyclovir dose used in primary episodes of genital herpes was $200 \mathrm{mg} 5$ times daily for 5 days in $96(86 \%)$ of respondents. Eleven physicians (10\%), reported using doses of at least double the data sheet recommended dose, and in one case, 4 times this dose. Two (2\%) physicians reported using doses less than the recommended daily dose. Thirty four $(30 \%)$ physicians reported the prescription of acyclovir cream for primary attacks, with no difference between the sexes, but more experienced physicians were more likely to prescribe topical treatment; physicians with more than 20 years experience $10 / 23$ (43\%), $10-20$ years $19 / 56(34 \%)$, less than 10 years $4 / 25(16 \%) \mathrm{p}<0.05$.

The most common additional intervention in primary attacks of herpes was counselling, with $86(77 \%)$ of physicians recommending this. The gender or experience of the physicians was not associated with provision of counselling.

\section{Management of individual recurrences}

Interventions in individual genital herpes recurrences are listed in table 1 . No significant differences with physicians gender or length of experience were identified.

\section{Suppressive acyclovir}

One-hundred three physicians responded to a question asking about the number of attacks of genital herpes per year influencing prescriptions of suppressive acyclovir, and their responses are shown in the figure.

Apart from the annual attack rate, physi-

Table 1 Management of individual genital herpes recurrences

\begin{tabular}{lc}
\hline Simple only (for example Saline bathing) & $39(35 \%)$ \\
Acyclovir cream & $21(19 \%)$ \\
Simple and/or acyclovir cream & $15(13 \%)$ \\
Oral acyclovir and/or cream & $13(12 \%)$ \\
Simple and/or oral acyclovir & $9(8 \%)$ \\
Oral acyclovir & $6(5 \%)$ \\
$\begin{array}{l}\text { Simple/acyclovir cream/tablets } \\
\text { Unanswered }\end{array}$ & $4(4 \%)$ \\
Therefore, 53 (47\%) regularly use & $5(4 \%)$ \\
acyclovir cream & either alone or \\
oral acyclovir & in combination \\
\hline
\end{tabular}

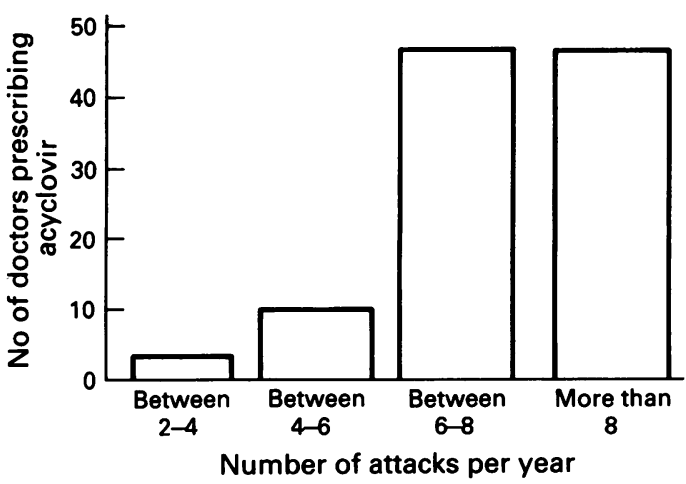

Figure Number of doctors prescribing acyclovir.

cians were asked to indicate other factors which would influence whether suppressive therapy was prescribed. These are illustrated in table 2. Significant differences were found between physicians' experience and three factors, HIV antibody status, patient anxiety and patients who were entering new relationships. Prescribing for HIV positive patients was more likely by physicians with fewer years in the speciality while the opposite was true for patient's anxiety. Physicians with more than 20 years experience were the least likely to prescribe when patients were starting a new relationship.

Questioned as to why suppressive therapy was prescribed, the most frequent response was to decrease psychological morbidity caused by recurrent herpes $(70(62 \%)$ of respondents) considerably more than those who prescribed principally to reduce multiple relapses $28(25 \%)$, or because of attacks interfering with normal life 12 (11\%). Physicians' gender or duration of experience did not influence these responses.

Duration of prescribing suppressive therapy is shown in table 3, with no differences by gender or experience of physician being found. Most physicians monitor patients on suppressive therapy at three monthly intervals $54(48 \%)$, or monthly intervals $38(34 \%)$. Most monitor with clinical history alone 79 $(71 \%)$, only $22(20 \%)$ using haematological and/or biochemical monitoring.

\section{Departmental policy}

Forty eight $(43 \%)$ of respondents stated that their department had a written policy on the management of genital herpes. Clinics appeared to be evenly divided as to whether only consultants 48 (43\%), or all doctors, 55 $(46 \%)$ could initiate prescriptions for continuous suppressive acyclovir. Only six physicians $(5 \%)$ reported pharmacy guidelines regarding prescriptions of acyclovir, but 13 physicians $(12 \%)$ reported financial stipulations relating to long term prescriptions.

\section{Referral to self-help groups}

Forty five physicians (40\%) report referring patients to self-help groups. This was not affected by the experience of the physician. Women were more likely to refer $13 / 25$ $(52 \%)$ compared with $28 / 76(37 \%)$ men, but this difference was not significant. Referrals 
Table 2 Factor influencing suppressive therapy vs physician experience

\begin{tabular}{llllll}
\hline Factor & $\begin{array}{l}\text { Overall } \\
(\%)\end{array}$ & $\begin{array}{c}<10 \text { years } \\
N=25\end{array}$ & $\begin{array}{c}10-20 \text { years } \\
N=56\end{array}$ & $\begin{array}{l}>20 \text { years } \\
N=23\end{array}$ & p Value \\
\hline HIV + & $86(77)$ & $23(92)$ & $40(71)$ & $16(70)$ & $<0.05$ \\
Anxiety & $47(42)$ & $7(28)$ & $29(52)$ & $10(43)$ & $<0.05$ \\
Patient's request & $30(27)$ & $5(20)$ & $17(30)$ & $6(26)$ & NS \\
New relationship & $20(18)$ & $5(20)$ & $12(21)$ & $1(4)$ & $<0.05$ \\
Partner without herpes & $5(4)$ & $1(4)$ & $3(5)$ & 0 & NS \\
\hline
\end{tabular}

were mainly to the Herpes Association 28 $(62 \%)$ and the associated Herpes Helpline 5 $(11 \%)$. Six (13\%) physicians reported having local self-help groups to which they referred patients.

\section{Does patient's gender affect coping?}

Physicians were asked if male patiients coped better with genital herpes than females and the overall response was equally dividied: $\mathbf{5 0}$ $(45 \%)$ yes, $38(34 \%)$ no, and $10(9 \%)$ equal. However, a significant difference was seen when the gender of respondents was taken into account: male physicians were significantly more likely to agree with this proposition than female physicians-41/76 (54\%) compared with $6 / 25(24 \%)(p<0.01)$. The duration of experience did not produce any signficant difference to this response.

\section{Discussion}

This survey gives an overview of the management of genital HSV infections by genitourinary physicians. The $62 \%$ response rate to this study was relatively low but this is not uncommon with postal surveys, including a number recently published. We believe the responses illustrate a reliable cross-section of genitourinary practice. The development of acyclovir has made a considerable impact on this condition and most physicians are confortable using this drug. However, the responses to the questionnaire illustrate that for both acute attacks and continuous suppressive therapy some of the differences in management are influenced by the physicians duration of clinical experience.

The finding that the most experienced physicians are more likely to reserve oral acyclovir for severe attacks may have several possible explanations. It may be that these physicians have developed counselling skills which enable them to support the patients through mild attacks without recourse to medication. Topical acyclovir is more popular with this group of physicians and this is possibly being used in place of oral therapy for less severe attacks. Furthermore, as acyclovir cream was launched before the oral prepara-

Table 3 How long is suppressive acyclovir used?

\begin{tabular}{lc}
\hline & Number of physicians \\
\hline$<2$ weeks & $2(2 \%)$ \\
$<1$ month & $3(3 \%)$ \\
$<2$ months & $7(6 \%)$ \\
$<4$ months & $13(12 \%)$ \\
$<6$ months & $31(28 \%)$ \\
$<1$ year & $29(26 \%)$ \\
Indefinitely & $12(11 \%)$ \\
\hline
\end{tabular}

tion some physicians may have established a prescribing pattern which has not significantly changed despite evidence that the systemic therapy is superior. ${ }^{4}$ The use of "self administered" topical treatment is common in genitourinary clinics (for example for candidiasis) and this may influence this preference for topical acyclovir. Finally, the cost of oral treatment exceeds the cost of topical therapy and this may also affect prescribing habits.

In contrast, more recently trained physicians may not be familiar with systemic acyclovir, as their training has often included the use of high dose regimes of this drug in HIV sero-positive patients. Doctors perception of the severity of genital herpes may vary with the duration of experience; ${ }^{5}$ the younger groups practice may be more influenced by recent publications about the psychological morbidity of genital HSV. ${ }^{26}$

A high percentage of consultants recognise the importance of early counselling in primary genital herpes. Recent information presented to a symposium stressed the importance of discussing with patients the implications of this condition. ${ }^{7}$ However, no evaluation has been performed as to whether basic information and support provided by the physician, or more specialised counselling is superior.

As $27 \%$ of physicians surveyed would prescribe for a patient with genital HSV "requesting acyclovir," this suggests that well informed patients, aware of the availability of suppressive therapy, are more likely to have such therapy prescribed for them. As patients become better informed, often via self-help groups, and the health service becomes increasingly consumer lead, the percentage of physicians prescribing suppressive acyclovir at a patients request may well increase.

The fact that the majority of practitioners prescribing suppressive therapy did not use any biochemical or haematological monitoring is evidence of the perceived low toxicity of this drug, as demonstrated by several studies. $^{89}$ The five per cent of physicians who responded that they would prescribe suppressive therapy for less than one month, may have misunderstood what was implied by this term and may not be familiar with this mode of acyclovir usage.

Cost may be an important factor in the decision to prescribe suppressive therapy. ${ }^{3}$ We found financial restraints to be uncommon at present, but an increase in departmental fundholding may change this. Some practioners indicated on our questionnaire that the patients who are on suppressive therapy are asked to request repeat prescriptions from their general practioner after the initiation of suppressive therapy in the genitourinary clinic, this has implications for patient care and in particular confidentiality.

There are a number of studies showing differences between men and women in regard to illness and behaviour. ${ }^{10}$ It may be that illness is less socially acceptable in men, and men may minimise their symptoms or distress compared with women. On the other hand, doctors may identify with patients of the 
same sex as themselves, and attribute greater ability to cope as a result to those more like themselves. Physicians gender might therefore have an indirect influence on prescribing habits, although we did not assess this in the questionnaire.

This survey has documented a range of -different management strategies for genital herpes by consultant genitourinary physicians. This is not surprising considering the variation amongst physicians in the management of other sexually transmitted diseases such as chlamydial cervicitis, 11 where many physicians use different treatment and have different follow-up policies for test of cure. Our study not only shows such differences but also delineates the effect that the experience and gender of the physician has upon them. Further work on the doctor/patient interaction in genital herpes and other genitourinary conditions to explore this relationship is warranted.
1 Goldmeier D, Johnson A, Byrne $M$, Barton $S$ Psychosocial implications of recurrent genital herpes Psychosocial implications of recurrent genital herpes
simplex infection, Genitourin Med 1988;64:327-30.

2 Drob S, Loemer M, Lifshutz H Genital herpes: The psyDrob S, Loemer M, Lifshutz H Genital herpes: The psy-
chological consequences. Br $\mathcal{F}$ Psychol. 1985;58:307-15.

3 Mindel A Reluctance to prescribe suppressive oral acyclovir for recurrent genital herpes. Lancet 1990;335 1107.

4 Kinghorn G R, Abeywickcreme I, Jeavons $M$ et al Efficiency of combined treatment with oral and topical acyclovir in first episode genital herpes. Genitourin Med 1986;62:186-8.

5 Bierman S M Recurrent genital herpes simplex infection. A trivial dislumer. Arch. Dermatol. 1985;121:513-7.

6 Longo D J, Clum G A Psychological factors affecting genital herpes recurrences: Linear vs mediating models. $\mathcal{f}$ ital herpes recurrences: Linear
Psychosom. Res 1989;33:161-6.

7 Hodson P Counselling - vital part of herpes management-Viewpoint genital herpes management 1991 ment-Viewpoint genital herpes management 1991
(Colwood House Medical Publications).

8 Mindel A, Faherty A, Hindey D et al. Prophylactic oral acyclovir in recurrent genital herpes. Lancet 1984;ii: $57-9$

9 Kaplowitz L G, Baker D, Gelb L et al Prolonged continuous acyclovir treatment of normal adults with frequently recurring genital herpes simplex infection. $¥ A M A$ 1991;265:747-51.

10 Briscoe M E Sex differences in perception of illness and expressed life satisfaction. Psychol Med 1978;8:339-45.

11 Radcliff $\mathrm{K} \mathrm{W}$, Rowen D, Mercey D E, Bingham J S Survey of the management of Chlamydia trachomatis Survey of the management of Chlamydia trachomatis
infection of the cervix. Genitourin Med 1991;67:41-3. 